\title{
Free Flap Reconstruction after Complications of Total Ankle Arthroplasty: Case Series and Review of the Literature
}

\author{
Jocelyn Lu, BS ${ }^{1}$ Tammer Elmarsafi, DPM, MBBCh ${ }^{2}$ John S. Steinberg, DPM ${ }^{3}$ Paul J. Kim, DPM, MS ${ }^{3}$
} Christopher E. Attinger, MD ${ }^{3}$ Paul Cooper, MD Karen K. Evans, MD ${ }^{3}$

\footnotetext{
${ }^{1}$ Department of Plastic Surgery, Georgetown University School of Medicine, Washington, District of Columbia

2 Diabetic Limb Salvage, Department of Plastic Surgery, MedStar Georgetown University Hospital, Washington, District of Columbia

${ }^{3}$ Department of Plastic Surgery, MedStar Georgetown University

Hospital, Washington, District of Columbia

${ }^{4}$ Department of Orthopedic Surgery, MedStar Georgetown University

Hospital, Washington, Districy of Columbia
}

J Reconstr Microsurg Open 2018;3:e13-e20.

\begin{abstract}
Address for correspondence Karen K. Evans, MD, Center for Wound Healing and Hyperbaric Medicine, MedStar Georgetown University Hospital, 3800 Reservoir Road NW, Washington, DC 20007 (e-mail: Karen.K.Evans@gunet.georgetown.edu).
\end{abstract}

\begin{abstract}
Keywords

- microsurgical free flap

- total ankle arthroplasty

- limb salvage

Background Postoperative complications of total ankle arthroplasty (TAA) include anterior surgical site dehiscence, hardware failure, infection, and amputation. Early intervention with free flap may provide TAA salvage. We report the largest series of failed TAA require microsurgical free tissue transfer, identify risk factors, and examine the long-term post-free flap outcomes.

Materials and Methods This is a case series of consecutive patients from a single institution dedicated to limb salvage. Inclusion criteria included patients who underwent TAA with complications related to the index surgery and underwent microsurgical free tissue transfer. Nine patients were identified within the inclusion parameters.

Results Patients presented with osteomyelitis 4 (44\%), soft tissue infections 4 (44\%), and wound dehiscence 1 (11\%) following TAA. Three (33\%) radial forearm free flaps and six $(67 \%)$ anterolateral thigh flaps were used, with a $100 \%$ microsurgical success rate. Preoperative angiography revealed six (67\%) patients with anterior tibial artery occlusion at the level of the ankle or below. Patients required an average of $2.7 \pm 1$ (range 1-4) operative débridements prior to free flap, with successful flap outcome and return to full weight bearing status in nine $(100 \%)$ patients. The mean long-term lower extremity functional scale score was 62 out of 80 points.

Conclusion Microsurgical free tissue transfer is an effective and favorable strategy to attain a stable soft tissue envelope for patients presenting with surgical site complications following TAA. We recommend early involvement with plastic surgery and endovascular angiography to evaluate the integrity of the anterior tibial artery.
\end{abstract}

In patients suffering from debilitating ankle arthritis, total knee arthroplasty (TAA) has become an increasingly popular choice over ankle arthrodesis due to its comparable outcomes and improved gait and range of motion. ${ }^{1,2}$ Since the first TAA in $1970,{ }^{3}$ increased surgeon experience and technological advancements have led to improved patient outcomes and survivability. ${ }^{2}$ As TAA is relatively new, long-term outcomes have not completely been elucidated. One meta- received

December 27, 2017

accepted after revision

February 22, 2018
DOI https://doi.org/

10.1055/s-0038-1641733. ISSN 2377-0813.
Copyright $\odot 2018$ by Thieme Medical Publishers, Inc., 333 Seventh Avenue, New York, NY 10001, USA. Tel: +1(212) 584-4662.
License terms

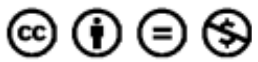


analysis showed TAA survivorship of $70 \%$ to $98 \%$ at 3 to 6 years and $80 \%$ to $95 \%$ at 8 to 12 years, ${ }^{2}$ but there is still much to uncover about the incidence and outcomes of complications after TAA.

The increased use of TAA has brought awareness of potential complications associated with ankle arthroplasty. ${ }^{4,5}$ Two of the most significant complications of TAA that have the greatest impacts on quality of life and patient function outcomes include postoperative wound breakdown and infection. ${ }^{6-8}$ Dehiscence for the anterior approach incision is the most common soft tissue complications following TAA, with reports of $8.5 \%$ to $28 \%$ of patients after ankle arthroplasty. ${ }^{6,7,9}$ Though the anterior incision offers convenient access to the ankle joint, this incisional approach can lead to vascular damage, causing wound healing issues, dehiscence, and necrosis of bony and soft tissues..$^{5,6,8}$ Minor wounds, small dehiscence, and superficial infections can quickly seed into deeper tissues, increasing risk of hardware removal and excision of infected tissue.

This poses a challenge, as blood flow to the distal extremity can be tenuous in this population, and adequate soft tissue coverage of the mobile ankle can be difficult. Failure to reestablish structural integrity and provide soft tissue coverage can be debilitating, leading to ankle arthrodesis or limb amputation. To avoid these unwanted consequences, microsurgical free flap can serve as an effective means to restore adequate soft tissue to the anterior ankle, as incorporation of free tissue transfer is known to enhance limb salvage outcomes. ${ }^{10}$ Understanding the morbidity associated with TAA complications, patient selection, preoperative planning, patient optimization, and adequate postsurgical surveillance all play an important role for positive outcomes. ${ }^{8,11}$

There is a paucity of literature regarding complications related to ankle replacement and its sequelae, particularly when these complications require complex reconstructive efforts. The purpose of this article is to evaluate the complications of TAA that require free tissue transfer, and review outcomes following free flap reconstruction for failed TAA.

\section{Materials and Methods}

This article is an institutional review board (IRB)-approved retrospective case series from a single institution. Patients were selected from a secure database of all free tissue transfers from January 2011 to December 2016, performed by one plastic surgeon (K.K.E). Inclusion criteria included patients who had previously undergone TAA, suffered complications related to the index surgery, and required free flap reconstruction. Nine TAA patients were eligible for analysis.

Operative records, imaging, and clinic notes were reviewed to define indications for TAA, complications secondary to TAA, lower extremity angiographic findings, type of free flap, and microsurgical technique. Dates and number of operations were recorded to analyze intervention type and timeliness. Outcomes followed included patient survival, flap survival, length of follow-up, complications after free flap, and status of TAA at last follow-up. Lower extremity functional scale questionnaire (LEFS) was administered postoperatively. Due to the retrospective nature of this series, preoperative LEFS was not able to be obtained.

All patients presented with complications secondary toTAA, prompting a standard management algorithm developed by the senior author (-Figs. 1 and 2). This included a biomechanical evaluation, vascular examination, soft tissue envelope assessment, laboratory tests, and lower extremity imaging. Labs included at minimum serial complete blood count with differential, C-reactive protein, erythrocyte sedimentation rate, prealbumin, glycated hemoglobin, international normalized

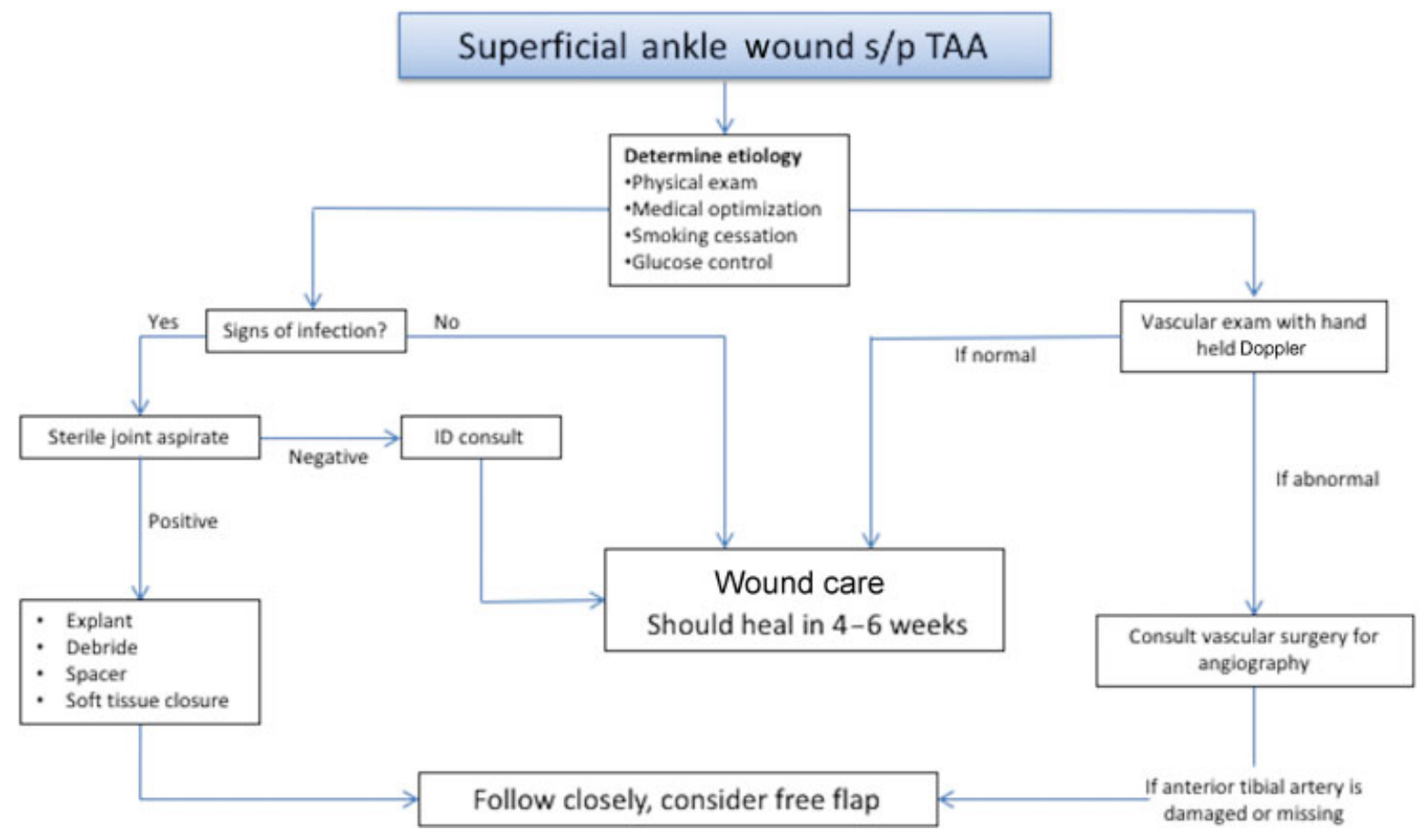

Fig. 1 Superficial wound algorithm after total ankle arthroplasty (TAA). 


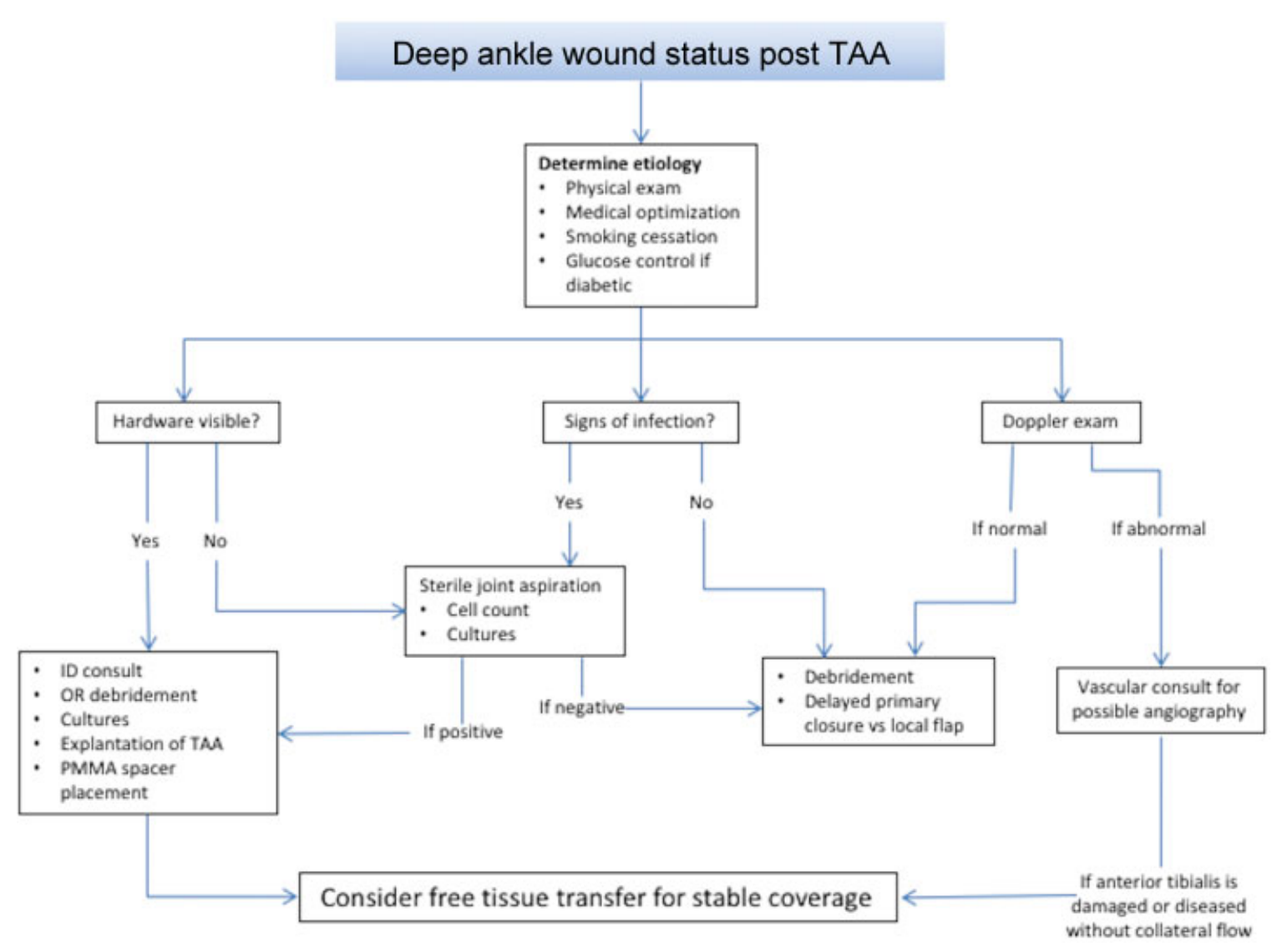

Fig. 2 Deep wound algorithm after total ankle arthroplasty (TAA).

ratio, and complete metabolic panel. All patients received plain radiographs of the affected ankle and advanced imaging as needed on a case-by-case basis. All patients received a vascular evaluation with handheld Doppler screening and angiography, with intervention as needed as part of the comprehensive evaluation for surgical planning.

Specialty consultations included infectious disease, internal medical, orthopedic surgery, plastic surgery, vascular surgery, rheumatology, and physical therapy. All patients were medically optimized and risk stratified prior to treatment. Each patient required operative débridement during which deep tissue cultures and bone biopsies were obtained. When appropriate, negative pressure wound therapy with instillation was utilized between each surgical débridement.

The treatment algorithm was based on involvement of the ankle joint. If the ankle capsule was not violated, sterile technique was used to obtain a joint aspirate. If the wound extended into the joint and/or the hardware was exposed, all hardware was explanted, followed by the placement of an antibiotic eluting polymethylmethacrylate (PMMA) cement spacer. The primary goal for serial surgical débridement was to excise grossly infected, devitalized tissue and to remove biofilm. Cultures and sensitivities were utilized to determine parenteral antibiotic choice, confirm eradication of infection, and permit initial closure.

The timing for soft tissue reconstruction was a critical factor in the management protocol. Guided by deep tissue cultures and the infectious disease team, intravenous anti- biotics were given for 6 weeks. In patients with antibiotic spacers, an "antibiotic washout" period of 4 weeks followed to monitor for recurrent infection. At the end of the 4-week period, a joint aspirate was performed. If positive, the sequence was repeated until culture results from the joint aspirate were negative, at which point final osseous reconstruction was completed by orthopedic surgery. The selection of free flap was based on multiple host factors, including aesthetic and functional outcomes.

A standard dangle protocol was utilized with ambulation on the contralateral limb on the 7th postoperative day. The operative limb was kept nonweight bearing based on the orthopedic surgeon's recommendations. Passive and active range of motion was initiated 6 weeks post-free flap reconstruction.

\section{Results}

\section{Patient Demographics}

Six (67\%) patients were male and three (33\%) were female. Mean age was $69 \pm 6$ years (range 61-80 years) and mean body mass index was $27.1 \pm 4(23.1-36.5)$. Patient comorbidities included hypertension 5 (56\%), peripheral arterial disease 5 (56\%), history of smoking 4 (44\%), diabetes mellitus 3 (33\%), and hyperlipidemia 3 (33\%) (-Table 1). Eight out of nine cases $(89 \%)$ presented with infection. Of the eight infections, 4 (44\%) were diagnosed with osteomyelitis, and $4(44 \%)$ were limited to soft tissue. The remaining patient (11\%) presented with ischemic necrosis and eschar over the 


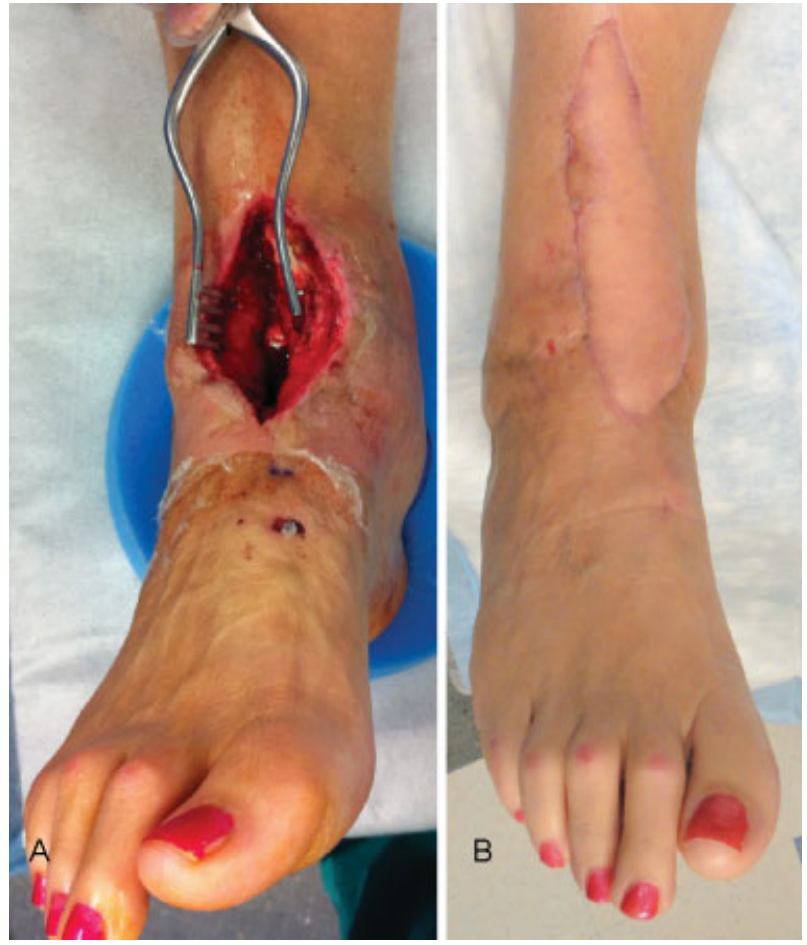

Fig. 3 (A) Preoperative view of infected total ankle wound with exposed hardware. (B) Successful radial forearm free flap to the ankle 6 weeks postoperatively.

Table 1 Patient demographics

\begin{tabular}{|l|l|l|}
\hline & $N(\%)$ & Mean \\
\hline Age & & $69 \pm 5.9(61-80)$ \\
\hline BMI & & $27.6 \pm 4.1(23.4-36.5)$ \\
\hline Male & $5(66.7 \%)$ & \\
\hline Female & $3(33.3 \%)$ & \\
\hline DM & $3(33.3 \%)$ & \\
\hline HTN & $5(55.6 \%)$ & \\
\hline HLD & $3(33.3 \%)$ & \\
\hline Renal & $0(0 \%)$ & \\
\hline COPD & $1(11.1 \%)$ & \\
\hline PAD & $5(55.6 \%)$ & \\
\hline History of smoking & $5(55.6 \%)$ & \\
\hline
\end{tabular}

Abbreviations: BMI, body mass index; CKD, chronic kidney disease; DM, diabetes mellitus; ESRD, end stage renal disease; HLD, hyperlipidemia; HTN, hypertension; PAD, peripheral arterial disease.

Table 2 Indications for TAA, complications, and time course anterior incision after TAA without signs of infection (-Table 2). The mean number of TAA surgeries was $2.3 \pm 0.4$ (range $1-4$ ). The mean time to post TAA complication was $6.1 \pm 3.6$ months (range $0.1-33.6$ ). The mean time to free flap intervention after TAA complication was $1.9 \pm 3.1$ months (range 0.1-9.7 months).

\section{Management of Joint}

Five (55\%) of the original implants were salvaged without removal. Four (44\%) patients required explant of the TAA and placement of a temporary PMMA spacer due to positive joint cultures and osteomyelitis. Three of these four (75\%) eventually underwent final TAA reimplantation once they were deemed infection free. The last patient still had a PMMA spacer with plans for TAA in the future. Overall, eight patients had implanted TAA, and one patient had PMMA spacer in place at most recent follow-up.

\section{Vascular Interventions}

All patients (100\%) received anatomic confirmation of vascular flow of the tibial vessels. Endovascular angiograms were performed for eight (89\%) patients before free tissue transfer to assess patency and flow of blood to the foot and ankle. One (11\%) patient had a computed tomography angiogram to confirm adequate blood flow and did not require further testing or intervention. Six (67\%) patients had a distally occluded anterior tibial artery at or just below the level of the ankle. The remaining three (33\%) patients had normal angiograms with three vessel run-off. Two (22\%) patients required angioplasty of the anterior tibial artery prior to free flap.

\section{Surgical Technique}

Patients underwent an average of $2.7 \pm 1$ (range 1-4) débridement prior to free flap. There were three (33\%) radial forearm free flaps (RFFF) and six (67\%) anterolateral thigh flaps (ALT). Microsurgical technique consisted of seven (78\%) anterior tibial anastomoses and two (22\%) posterior tibial artery anastomoses. Of the seven anterior tibial artery anastomoses, four (57\%) were end-to-side and two (29\%) were end-to-end. The anastomosis occurred proximal to the area of occlusion outside the zone of injury. Both posterior tibial artery anastomoses were end-to-side (100\%). All flaps had a successful microsurgical outcome (100\%).

\section{Outcomes}

There was one (11\%) early post flap complication-a patient developed a postoperative hematoma at the recipient site of his ALT flap 9 days after microvascular free tissue transfer. There were no donor site morbidities or difference in outcomes between RFFF and ALT flaps (- Table 3). Intermediate

\begin{tabular}{|l|l|l|l|l|l|l|}
\hline & \multicolumn{3}{|l|}{ Indication for TAA } & \multicolumn{4}{l|}{ Presenting complication } \\
\hline & Post-traumatic OA & Degenerative OA & Soft tissue infection & Infected hardware & OM & Gangrene \\
\hline$N(\%)$ & $3(33.3 \%)$ & $6(66.7 \%)$ & $3(33.3 \%)$ & $1(11.1 \%)$ & $4(44.4 \%)$ & $1(11.1 \%)$ \\
\hline
\end{tabular}

Abbreviation: OA, osteoarthritis; OM, osteomyelitis; TAA, total ankle arthroplasty. 
Table 3 Types of free flaps and outcomes

\begin{tabular}{|l|l|l|l|l|l|}
\hline & $N(\%)$ & Flap success & Ambulatory after flap & FWB after flap & $\begin{array}{l}\text { LEFS } \\
\text { Mean } \pm \text { SD (range) }\end{array}$ \\
\hline RFFF & $3(33 \%)$ & $9(100 \%)$ & $9(100 \%)$ & $9(100 \%)$ & $56 \pm 2.8(54-58)$ \\
\hline ALTF & $6(67 \%)$ & $9(100 \%)$ & $9(100 \%)$ & $9(100 \%)$ & $65 \pm 15.4(44-80)$ \\
\hline
\end{tabular}

Abbreviations: ALT, anterolateral thigh flap; FWB, full weight bearing; LEFS, lower extremity functional scale; RFFF, radial forearm free flap; SD, standard deviation.

post flap outcomes at 3 months in our cohort have shown that all 9/9 patients (100\%) were successfully transitioned to full weight bearing after free tissue transfer and had no flap compromise (-Figs. 3-7). Long-term follow-up at an average of $14.5 \pm 19.3$ months (range $0-57.6$ months) was obtained via medical records or direct patient contact. Long-term functional outcomes were evaluated by utilizing the LEFS which was obtained from 6 patients at an average of 23.3
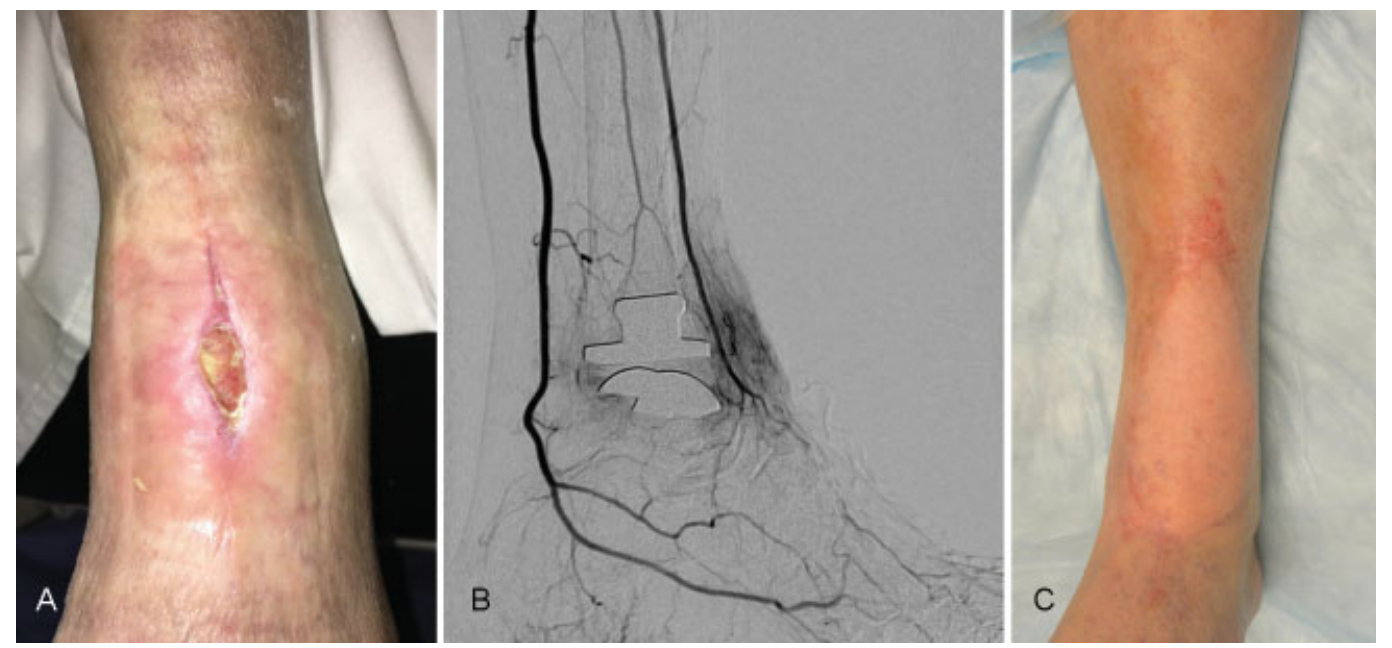

Fig. 4 (A) Preoperative image of a superficial anterior ankle wound after total ankle arthroplasty. Wound was present for 2 months with minimal healing. (B) Angiogram was obtained after a handheld Doppler exam revealed minimal flow in the anterior tibial artery. (C) Three-month postoperative image status post anterior lateral thigh free flap to the ankle. Sterile joint aspirate as well as intraoperative wound cultures is negative; thus, the total ankle prosthesis was salvaged and free tissue transfer was used to cover the defect.
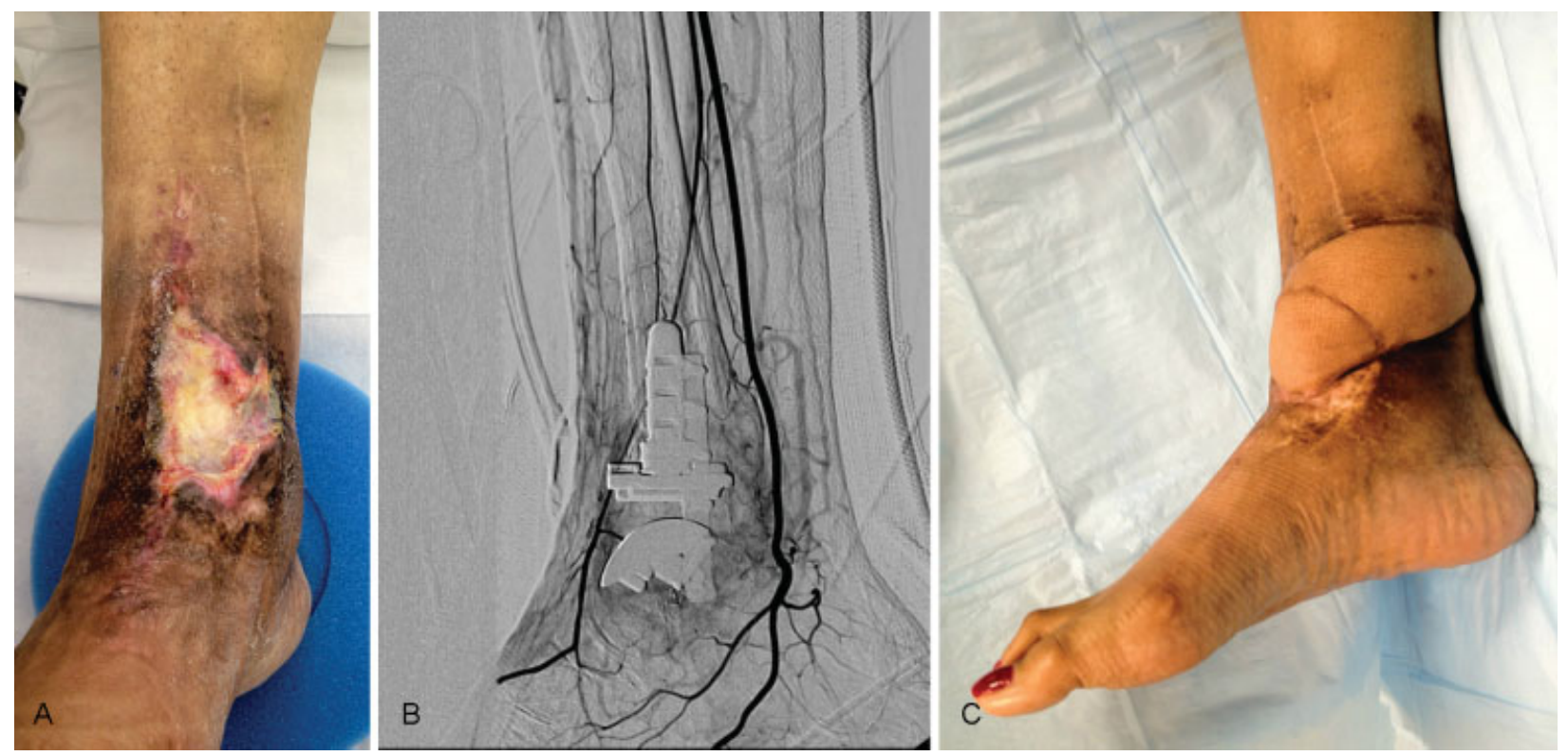

Fig. 5 (A) Anterior ankle defect status post total ankle arthroplasty. Patient underwent attempts at skin grafting, HBO, and local tissue mobilization; however, her wound was still persistent for 5 months. (B) Angiogram revealed dominant flow in the PT artery with minimal anterior tibial artery flow. (C) A radial forearm free flap was harvested and an end to side anastomosis was performed to the posterior tibial artery. Patient underwent eventual successful reimplantation with new arthroplasty. 


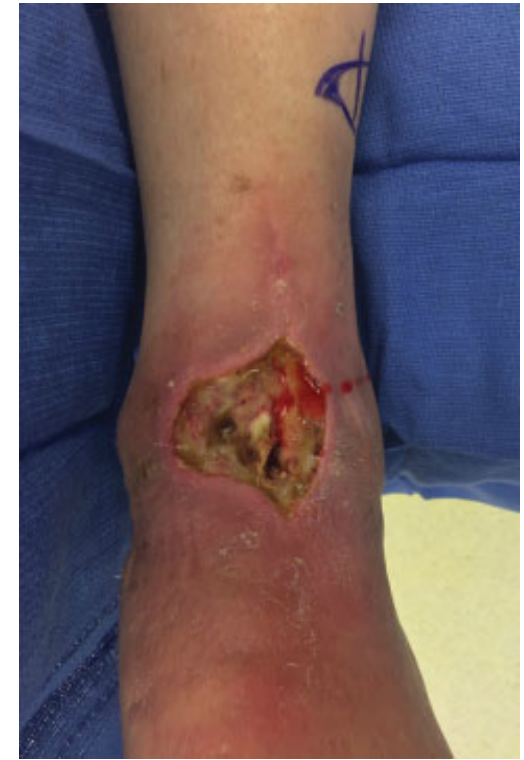

Fig. 6 Deep ankle wound with obvious cellulitis and drainage status post total ankle arthroplasty.

months after free flap. LEFS scores showed an average of $62 \pm 12.9$ out of 80 (range $44-80$ ).

One $(11 \%)$ patient underwent an elective ankle arthrodesis and subsequent below knee amputation 53.8 months after free flap secondary to pain. These operations were performed at an outside hospital. In addition, there was one (11\%) patient death at 83 years of age unrelated to his lower extremity.

\section{Discussion}

Wound dehiscence after TAA is not an uncommon phenomenon, and can lead to serious complications with a prolonged and costly hospital course. ${ }^{2,5,6,8}$ These complications oftentimes lead to amputation due to the significant morbidity associated with multiple operations on a weight bearing joint and infection of hardware. Our current study is the largest series with the longest follow-up demonstrating that free flap reconstruction can be a successful and expeditious method to cover defects following TAA complications. All flaps survived and the ultimate outcomes of limb salvage were favorable as represented by a high mean LEFS score of $62 \%$ and $100 \%$ ambulatory rate. The LEFS scoring system tracks changes in lower limb function based on a 21 -item questionnaire, the sum of which corresponds to a numerical scale from 0 to 80 , and a correlated descriptive functional stage of 1 to 5 . A score of 62 falls between an independent community ambulator and an active community ambulator. ${ }^{12,13}$

A literature search revealed only two articles that describe the use of free tissue transfer following wound breakdown after TAA. ${ }^{14,15}$ A case series by Hallock describes five patients who suffered delayed wound breakdown after TAA, and showed free flap reconstruction to be an effective method to salvage arthroplasty. ${ }^{15}$ Patients presented with complications of wound healing, and the average time from presentation to free flap was 9.8 weeks. Though all five patients in the study had successful outcome including hardware salvage and the ability to ambulate postoperatively, follow-up in these patients was at most 12 months. ${ }^{15}$ In addition to advocating for the use of free flap after TAA complications, we stress the importance of long-term follow-up in this patient population, as this can assess whether complex reconstruction in this patient population is warranted and durable.

The study by Gross et al discusses the relationship between time of intervention and outcomes a cohort of 11 local flaps and 8 free flaps after TAA. They reported a mere 13.1 weeks between presentation and flap. But even with such rapid

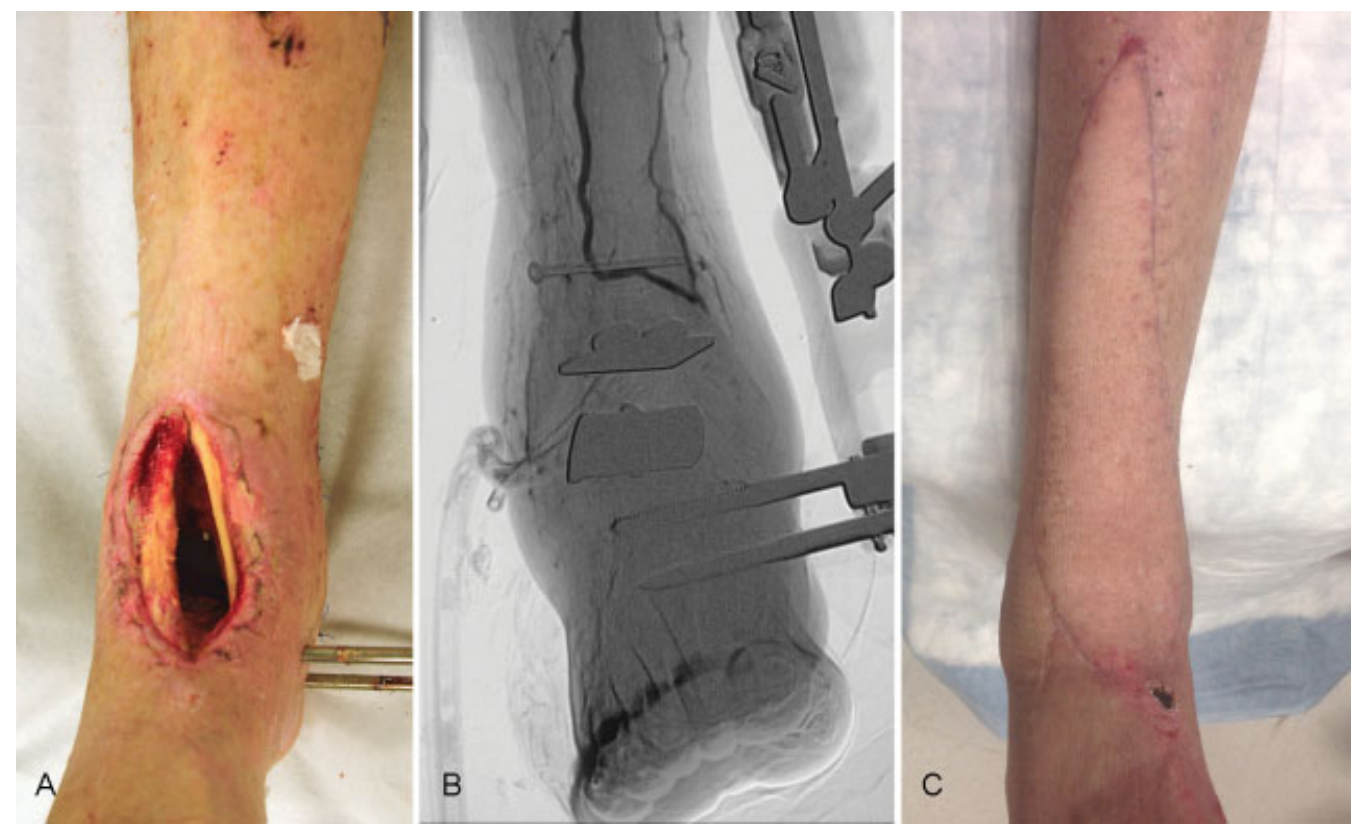

Fig. 7 (A) Preoperative image of a patient with an infected total ankle arthroplasty status post explantation and placement of polymethylmethacrylate spacer and external fixator. (B) Angiography image showing ischemia in the anterior tibial angiosome with dominant posterior tibial artery. (C) Patient underwent successful anterolateral thigh flap coverage and later exchange to permanent total ankle prosthetic. 
intervention, they still had a $21 \%$ flap failure rate, and a $10.5 \%$ below knee amputation rate. Failures were due to chronic nonhealing wounds or flap necrosis. It is important to note that the authors did not discriminate data from local and free flaps and thus comparison to our data may be difficult. Furthermore, the authors make no mention of vascular evaluation. These flap failures in the setting of early intervention point toward the importance of angiography and Doppler screening in planning process for successful free flap outcomes.

In our series, there was only one significant free flap-related complication in the immediate postoperative period-development of hematoma under the flap, which was thought to have been due to supratherapeutic anticoagulation from Coumadin given postoperatively as prophylaxis secondary to underlying atrial fibrillation. The hematoma was evacuated and the patient went on to have an uneventful recovery. After adjusting his medications, he suffered no further complications.

Early recognition of infection and dehiscence with early intervention may offer better long-term success and limb salvage rates. One study showed the interval from TAA to diagnosis of infection was on average of 18 months, with an amputation rate of $21 \%{ }^{16}$ Their mean follow-up was 55 months. Our patient series had a relatively acute presentation with mean of $6.1 \pm 3.6$ months (range 0.1-33.6) from TAA to presentation of infection. Our amputation rate was $11 \%$. However, in our series, this patient required an amputation 53.8 months after a successful free flap unrelated to infection. It is important to follow these patients for long term to analyze overall salvage rates. We hypothesize that delayed intervention leads to worse limb salvage outcomes. Early intervention and treatment of infection aim to minimize the extent of involved tissue and deeper structures, effectively mitigating soft tissue and bone loss as well as the arthroplasty.

The multidisciplinary team approach which includes vascular surgery is paramount for the overall success of this complex patient population. It was interesting to note that $67 \%$ of patients had a compromised anterior tibial artery which was either pre-existing or due to intraoperative injury at the time of the initial TAA. Without adequate blood flow, surgical site dehiscence, infection, and delayed healing can occur, leading to post-TAA failures. Preoperative angiography and adequate patient selection and screening are an imperative component of the TAA process and free flap planning. Patients who require TAA generally fall into an age category which puts them at increased cardiovascular risk and thus must be adequately assessed from a vascular perspective. Additionally, the incision for the TAA is both extensive and aggressive and the soft tissue manipulation during implantation can be unforgiving. These reasons should lower the threshold for endovascular angiography, particularly in those with a positive smoking history and/or diabetes. Furthermore, secondary healing or simpler methods of closure such as delayed primary closure may be less effective than free flap.

A free tissue transfer can replace compromised or watershed areas in the anterior tibial ischemic area and is the preferred method of closure over local flaps or other modalities. ${ }^{17,18}$ For the lower $1 / 3$ of the lower extremity, free flap reconstruction is the first choice due to the blood supply of local flaps. Local flaps in the distal extremity are based on secondary blood supply or reverse flow which are limited in this patient population. Other options for coverage in the distal $1 / 3$ are perforator propeller flaps which are also limited when there are areas of ischemia.

Patients in this series underwent either RFFF or ALT procedures. The choice between RFFF and ALT was decided based on functional and aesthetic outcomes. Flap bulk played a large role, as bulky flaps at the anterior ankle can be unsightly, and interfere with shoe and boot wear. ALT flaps were used if preoperative Allen's test was abnormal and preferentially used over RFFF if the thigh was thin. RFFF is a thin fasciocutaneous flap which replaces the anterior ankle defect with supple tissue and is used primarily if ALT flap is too bulky, especially in obese patients. ${ }^{19,20}$ The biggest disadvantage to the RFFF is the donor site morbidity, ${ }^{20}$ which in this study posed no complications. Muscle flaps such as gracilis flaps are also considered in this location but free fasciocutaneous flaps are equally successful and may offer less donor site morbidity. ${ }^{21,22}$

Patient outcomes including ambulation and weight bearing status of the nine patients show that free flap is indeed an effective method for salvage of TAA and reconstruction of soft tissue defects after TAA. In patients with osteomyelitis who required explant and a temporary antibiotic spacer, free flap was an effective method to heal the open wound and allow for a new TAA at a later date.

\section{Conclusion}

Complex soft tissue reconstruction of the ankle particularly after osseous and hardware infection requires a comprehensive evaluation and prompt management protocol. The anterior tibial artery is an at-risk vital structure susceptible to injury during TAA operation and reoperation, setting the stage for wound dehiscence and long-term complications. Doppler screening and angiographic evaluation are highly encouraged, and the multidisciplinary approach with plastic surgery and vascular surgery is vital to the successful outcome. Free flaps can serve as an effective early intervention for reconstruction of wounds after TAA complications by restoring the soft tissue envelope with adequately revascularized tissue. This allows the patients to re-engage in their functions of daily living. Long-term functional outcomes based on our data, despite the extensive medical and surgical interventions posed to the patients in our series, are promising. Further studies are encouraged to evaluate long-term outcomes in comparison to amputation.

\section{Funding}

None.

Conflict of Interest

None. 


\section{References}

1 Jiang JJ, Schipper ON, Whyte N, Koh JL, Toolan BC. Comparison of perioperative complications and hospitalization outcomes after ankle arthrodesis versus total ankle arthroplasty from 2002 to 2011. Foot Ankle Int 2015;36(04):360-368

2 Easley ME, Adams SB Jr, Hembree WC, DeOrio JK. Results of total ankle arthroplasty. J Bone Joint Surg Am 2011;93(15):1455-1468

3 Gougoulias N, Maffulli N. History of total ankle replacement. Clin Podiatr Med Surg 2013;30(01):1-20

4 Spirt AA, Assal M, Hansen ST Jr. Complications and failure after total ankle arthroplasty. J Bone Joint Surg Am 2004;86-A(06): $1172-1178$

5 Singh JA, Ramachandran R. Time trends in total ankle arthroplasty in the USA: a study of the National Inpatient Sample. Clin Rheumatol 2016;35(01):239-245

6 MatsumotoT, Parekh SG. Use of negative pressure wound therapy on closed surgical incision after total ankle arthroplasty. Foot Ankle Int 2015;36(07):787-794

7 Raikin SM, Kane J, Ciminiello ME. Risk factors for incision-healing complications following total ankle arthroplasty. J Bone Joint Surg Am 2010;92(12):2150-2155

8 Kessler B, Sendi P, Graber P, et al. Risk factors for periprosthetic ankle joint infection: a case-control study. J Bone Joint Surg Am 2012;94(20):1871-1876

9 Whalen JL, Spelsberg SC, Murray P. Wound breakdown after total ankle arthroplasty. Foot Ankle Int 2010;31(04):301-305

10 Wettstein R, Schürch R, Banic A, Erni D, Harder Y. Review of 197 consecutive free flap reconstructions in the lower extremity. J Plast Reconstr Aesthet Surg 2008;61(07):772-776

11 Glazebrook MA, Arsenault K, Dunbar M. Evidence-based classification of complications in total ankle arthroplasty. Foot Ankle Int 2009;30(10):945-949

12 Binkley JM, Stratford PW, Lott SA, Riddle DL; North American Orthopaedic Rehabilitation Research Network. The Lower Extremity Functional Scale (LEFS): scale development, measurement properties, and clinical application. Phys Ther 1999;79(04): 371-383

13 Wang Y-C, Hart DL, Stratford PW, Mioduski JE. Clinical interpretation of a lower-extremity functional scale-derived computerized adaptive test. Phys Ther 2009;89(09):957-968

14 Gross CE, Garcia R, Adams SB, DeOrio JK, Easley ME, Nunley JA II. Soft tissue reconstruction after total ankle arthroplasty. Foot Ankle Int 2016;37(05):522-527

15 Hallock GG. The role of free flaps for salvage of the exposed total ankle arthroplasty. Microsurgery 2017;37(01):34-37

16 Patton D, Kiewiet N, Brage M. Infected total ankle arthroplasty: risk factors and treatment options. Foot Ankle Int 2015;36(06): 626-634

17 Hollenbeck ST, Woo S, Komatsu I, Erdmann D, Zenn MR, Levin LS. Longitudinal outcomes and application of the subunit principle to 165 foot and ankle free tissue transfers. Plast Reconstr Surg 2010; 125(03):924-934

18 Li X, Cui J, Maharjan S, Lu L, Gong X. Reconstruction of the foot and ankle using pedicled or free flaps: Perioperative flap survival analysis. Santanelli, di Pompeo d'Illasi F, ed. PLoS One 2016;11 (12): 00167827

19 Medina MA III, Salinas HM, Eberlin KR, et al. Modified free radial forearm fascia flap reconstruction of lower extremity and foot wounds: optimal contour and minimal donor-site morbidity. J Reconstr Microsurg 2014;30(08):515-522

20 Knott PD, Seth R, Waters HH, et al. Short-term donor site morbidity: a comparison of the anterolateral thigh and radial forearm fasciocutaneous free flaps. Head Neck 2016;38(Suppl 1): E945-E948

21 Demirtas Y, Neimetzade T, Kelahmetoglu O, Guneren E. Comparison of free muscle and perforator skin flaps for soft tissue reconstruction of the foot and ankle. Foot Ankle Int 2010;31(01):53-58

22 Paro J, Chiou G, Sen SK. Comparing muscle and fasciocutaneous free flaps in lower extremity reconstruction-does it matter? Ann Plast Surg 2016;76(Suppl 3):S213-S215 